VOL. $6(1972), 355-356$.

\title{
A remark on a theorem of Caradus
}

\section{J.A. Johnson}

It is shown how a result of S.R. Caradus on the approximation problem can be obtained from known theorems.

Terms used here are standard (see [1] or [3]).

Let $X$ denote a Banach space, $S$ its unit ball in the weak topology, and $X^{*}$ the dual of $X$. In [1], the following theorem is proved:

(I) If $X$ is reflexive and $X^{*}$ (considered as a subspace of the continuous scalar-valued functions $C(S)$ in the canonical way) is complemented in $C(S)$, then $X$ has the approximation property.

It is our purpose to point out that (I) is a corollary to some known theorems that yield the stronger conclusion (II) below.

(II) Let $X$ be any Banach space and $C(S)$ the space of bounded continuous functions on $S$. If $X^{*}$ is complemented in $C(\bar{S})$ then $X^{*}$, and therefore $X$, has the approximation property.

Proof. It is well known (see [2] for an elementary proof) that $C(K)$ has the approximation property for $K$ compact Hausdorff. Thus $C(S)$ does also (use the Stone-Čech compactification of $S$ ). Now it is an easy exercise to show that complemented subspaces inherit the approximation property. It is also well known that if $X^{*}$ has the approximation property, so does $X$ (see [3, Remark, p. 113]). This completes the proof.

[Added 6 December 1971]. After this note had been accepted for publication, it was pointed out to me by Professor Caradus that H.E. Lacey had also made the observation that a more efficient proof exists. His proof is somewhat different from mine, however. It shows that if $X$ is

Received 16 November 1971. 
reflexive and $X^{*}$ is complemented in $C(S)$ then $X$ is finite dimensional.

\section{References}

[1] S.R. Caradus, "The approximation problem for compact operators", BulZ. Austral. Math. Soc. 1 (1969), 397-401.

[2] Jesús Gil de Lamadrid, "On finite dimensional approximations of mappings in Banach spaces", Proc. Amer. Math. Soc. 13 (1962), 163-168.

[3] Helmut H. Schaefer, Topological vector spaces (The Macmillan Company, New York; Collier-Macmillan, London; 1966).

Oklahoma State University,

Stil Iwater,

Oklahoma,

USA. 